\title{
Functional Analyses of Glycyl-tRNA Synthetase Mutations Suggest a Key Role for tRNA-Charging Enzymes in Peripheral Axons
}

\author{
Anthony Antonellis, ${ }^{1}$ Shih-Queen Lee-Lin, ${ }^{1}$ Amy Wasterlain, ${ }^{1}$ Paul Leo, ${ }^{2}$ Martha Quezado, ${ }^{4}$ Lev G. Goldfarb, ${ }^{5}$ \\ Kyungjae Myung, ${ }^{3}$ Shawn Burgess, ${ }^{1}$ Kenneth H. Fischbeck, ${ }^{6}$ and Eric D. Green ${ }^{1}$ \\ ${ }^{1}$ Genome Technology Branch, ${ }^{2}$ Genetic Disease Research Branch, and ${ }^{3}$ Genetics and Molecular Biology Branch, National Human Genome Research \\ Institute, ${ }^{4}$ Laboratory of Pathology, National Cancer Institute, ${ }^{5}$ Clinical Neurogenetics Unit and ${ }^{6}$ Neurogenetics Branch, National Institute of Neurological \\ Disorders and Stroke, National Institutes of Health, Bethesda, Maryland 20892
}

Charcot-Marie-Tooth disease type 2D (CMT2D) and distal spinal muscular atrophy type V (dSMA-V) are axonal neuropathies characterized by a phenotype that is more severe in the upper extremities. We previously implicated mutations in the gene encoding glycyl-tRNA synthetase (GARS) as the cause of CMT2D and dSMA-V. GARS is a member of the family of aminoacyl-tRNA synthetases responsible for charging tRNA with cognate amino acids; GARS ligates glycine to tRNA ${ }^{\text {Gly }}$. Here, we present functional analyses of disease-associated GARS mutations and show that there are not any significant mutation-associated changes in GARS expression levels; that the majority of identified GARS mutations modeled in yeast severely impair viability; and that, in most cases, mutant GARS protein mislocalizes in neuronal cells. Indeed, four of the five mutations studied show loss-of-function features in at least one assay, suggesting that tRNAcharging deficits play a role in disease pathogenesis. Finally, we detected endogenous GARS-associated granules in the neurite projections of cultured neurons and in the peripheral nerve axons of normal human tissue. These data are particularly important in light of the recent identification of CMT-associated mutations in another tRNA synthetase gene [YARS (tyrosyl-tRNA synthetase gene)]. Together, these findings suggest that tRNA-charging enzymes play a key role in maintaining peripheral axons.

Key words: Charcot-Marie-Tooth disease; spinal muscular atrophy; peripheral neuropathy; axonopathy; tRNA synthetase; axonal translation

\section{Introduction}

Charcot-Marie-Tooth (CMT) disease comprises a heterogeneous group of inherited neurodegenerative disorders that affect the peripheral nerve. Although the overall presentation varies, the general clinical features of CMT include muscular weakness and atrophy in the distal extremities. An estimated 1 in 2500 individuals are affected with CMT, making it the most common inherited peripheral neuropathy (Skre, 1974). CMT can be divided into two classes based on motor nerve conduction velocities (MNCVs) (Dyck and Lambert, 1968). Patients with CMT type 1 (CMT1) have decreased MNCVs and evidence of demyelinating axons, whereas patients with CMT type 2 (CMT2) have normal MNCVs and no demyelination; as such, CMT1 is classi-

\footnotetext{
Received April 19, 2006; revised July 29, 2006; accepted Aug. 14, 2006.

This work was supported in part by the Intramural Research Program of the National Human Genome Research Institute, National Institutes of Health. A.A. was supported by a fellowship grant from the Charcot-Marie-Tooth Association. The National Institute of Neurological Disorders and Stroke Institutional Review Board approved al studies performed herein, and appropriate informed consent was obtained from all subjects. We thank the members of the CMT2D and dSMA-V families for their participation in this study. We also thank Bob Nussbaum and Ornit Chiba-Falek for providing SH-SY5Y cells, Darby Miller and Amy Avila for providing MN-1 cells, Paul Schimmel and Leslie Nangle for providing the RJT3/II-1 yeast strain, and Martine Behra for helpful discussions on microscopy.

Correspondence should be addressed to Dr. Eric D. Green, National Human Genome Research Institute, National Institutes of Health, 50 South Drive, Building 50, Room 5222, Bethesda, MD 20892. E-mail: egreen@nhgri.nih.gov. DOI:10.1523/JNEUROSCI.1671-06.2006

Copyright $\odot 2006$ Society for Neuroscience ～0270-6474/06/2610397-10\$15.00/0
}

fied as a demyelinating neuropathy and CMT2 as an axonal neuropathy.

Our previous genetic studies implicated glycyl-tRNA synthetase gene (GARS) mutations in two neurodegenerative diseases: CMT2D and distal spinal muscular atrophy type $\mathrm{V}$ (dSMA-V) (Antonellis et al., 2003). These two diseases are considered axonal neuropathies based on electrophysiological features and are distinguished by sensory loss seen in CMT2D but not dSMA-V patients (Antonellis et al., 2003; Sivakumar et al., 2005). Furthermore, CMT2D and dSMA-V patients tend to have more severe symptoms in the upper compared with lower extremities (Christodoulou et al., 1995; Ionasescu et al., 1996; Sambuughin et al., 1998; Antonellis et al., 2003; Sivakumar et al., 2005).

One possibility is that axonal neuropathies are caused by defects specific to the nerve cell axon. This notion is supported by the identification of other CMT-associated mutations in genes encoding proteins with a role in axonal transport or cytoskeleton maintenance (Mersiyanova et al., 2000; Zhao et al., 2001; Verhoeven et al., 2003; Evgrafov et al., 2004). GARS, however, is a ubiquitously expressed $\alpha 2$ homodimer responsible for charging tRNA molecules with glycine (Freist et al., 1996); this enzyme is essential for protein synthesis in all cells and tissues. Therefore, it is of interest to understand how defects in GARS can lead to the 
limited phenotype of peripheral axonal neuropathy that is more pronounced in the upper extremities.

In light of the identification of disease-associated mutations in multiple sites and within functional domains of the encoded protein, we hypothesized that GARS mutations cause impaired enzyme function and that peripheral nerve axons may be more sensitive than other cell types to impaired tRNA charging (Antonellis et al., 2003). Interestingly, this hypothesis is supported by the recent identification and characterization of YARS (tyrosyltRNA synthetase gene) mutations in patients with dominant intermediate CMT (Jordanova et al., 2006). Here, we describe functional analyses of disease-associated GARS mutations and present data that suggest an important role for tRNA-charging enzymes in the maintenance of axonal health.

\section{Materials and Methods}

GARS protein expression and purification. Wild-type GARS protein was expressed after cloning into pET21a(+) (Novagen, Madison, WI) using Escherichia coli BL21-CodonPlus(DE3)-RIL cells (Stratagene, La Jolla, $\mathrm{CA}$ ) and then purified under denaturing conditions using the ProBond purification system (Invitrogen, Carlsbad, CA) according to the instructions of the manufacturer. Nine $1 \mathrm{ml}$ fractions were eluted from the extraction column and concentrated on Microcon YM-10 columns (Millipore, Bedford, MA). The majority of the GARS protein was found to reside in the second $1 \mathrm{ml}$ fraction, as assessed by Western blot analysis using an anti-6XHis antibody (data not shown).

Northern blot analysis. Total RNA was isolated from cultured lymphoblastoid cell lines using the RNeasy Mini kit (Qiagen, Valencia, CA). Purified RNA (10 $\mu \mathrm{g})$ from each sample was loaded on $1 \%$ agarose gels, electrophoresed at $90 \mathrm{~V}$ for $4 \mathrm{~h}$, and transferred to Hybond-N nylon membranes (GE Healthcare, Piscataway, NJ) in $0.01 \mathrm{~N} \mathrm{NaOH} / 3 \mathrm{M} \mathrm{NaCl}$. A PCR product spanning nucleotides 1485-2200 of the GARS mRNA (GenBank accession number NM_002047) was generated from a fulllength cDNA clone (IMAGE 4109682) and radiolabeled with [ $\left.{ }^{32} \mathrm{P}\right] \mathrm{dCTP}$ using Ready-To-Go DNA labeling beads (GE Healthcare). After removal of unincorporated nucleotides, the probe was hybridized to the above Northern blots using ExpressHyb hybridization solution (Clontech Laboratories, Palo Alto, $\mathrm{CA}$ ) at $37^{\circ} \mathrm{C}$ for $1 \mathrm{~h}$. Membranes were washed twice in $2 \times \mathrm{SSC} / 0.1 \%$ SDS and then twice in $0.1 \times \mathrm{SSC} / 0.1 \% \mathrm{SDS}$, in each case for $40 \mathrm{~min}$ at room temperature. Hybridization results were captured using a Fujifilm FLA-5000 phosphoimager (Fujifilm, Stamford, CT). A probe for the $\beta$-actin gene was prepared and used in the same manner to assess differences in the total amount of RNA analyzed.

Real-time PCR. Allele-specific PCR primers for the wild-type (5'GCCTGGAGGAAACATGCCTG-3') and G240R (5'-GCCTGGAGGAAACATGCCTC-3') GARS mRNA were used in conjunction with a common reverse primer ( $5^{\prime}$-GAAGGTCTGCCACATTCTGG-3'). RNA was extracted as above, and reverse transcription was performed using poly-dT primers. Real-time PCR was performed using an iCycler iQ Real Time PCR Detection System (Bio-Rad, Hercules, CA) and measured by Syber green incorporation using the SYBR GreenER qPCR Supermix (Invitrogen). Allele copy number was assessed by including a vector of known copy number that contained either the wild-type or G240R GARS cDNA (see below). In parallel, a PCR product was generated from the $\beta$-actin gene to correct for variation in the generation of reverse transcription products. Similar analyses were performed to detect yeast glycyl-tRNA synthetase gene (GRS1) alleles. All primer sequences are available on request.

Western blot analyses. Cultured human lymphoblastoid cells were harvested and resuspended in $37.5 \mu \mathrm{l}$ of lysis solution containing $10 \mathrm{~mm}$ Tris- $\mathrm{HCl} / 1 \mathrm{~mm}$ EDTA/50 $\mathrm{mm} \mathrm{NaCl} / 1 \times$ protein-reducing agent (Invitrogen) and $12.5 \mu \mathrm{l}$ of loading buffer containing SDS (Invitrogen). After incubation at $95^{\circ} \mathrm{C}$ for $5 \mathrm{~min}$, the total protein lysate derived from $\sim 50,000$ cells was electrophoresed on $4-12 \%$ Bis-Tris gels (Invitrogen) at $125 \mathrm{~V}$ for $100 \mathrm{~min}$ in running buffer containing SDS (Invitrogen). Samples were then electroblotted onto nitrocellulose membranes at $25 \mathrm{~V}$ for $100 \mathrm{~min}$ in NuPAGE transfer buffer (Invitrogen). Membranes were blocked in $1 \times$ PBS $/ 0.05 \%$ Tween $20 / 5 \%$ nonfat dry milk for $18 \mathrm{~h}$ at $4^{\circ} \mathrm{C}$ and then incubated with a 1:5000 dilution of $1 \mu \mathrm{g} / \mu \mathrm{l}$ anti-GARS (see below), $1 \mu \mathrm{g} / \mu \mathrm{l}$ anti-6XHis (Invitrogen), or $1 \mu \mathrm{g} / \mu \mathrm{l}$ anti-actin (Sigma, St. Louis, MO) antibody in blocking solution for $1 \mathrm{~h}$ at room temperature. Membranes were washed three times in $1 \times$ PBS plus $0.05 \%$ Tween 20 , incubated with a secondary antibody conjugated to horseradish peroxidase [donkey anti-rabbit IgG (GE Healthcare) for anti-GARS and anti-actin, and rat anti-mouse IgG (Zymed Laboratories, South San Francisco, CA) for anti-6XHis] for $1 \mathrm{~h}$ at room temperature, washed three times as described above, and finally incubated with DAB substrate (Roche Diagnostics, Basel, Switzerland), according to the manufacturer's instructions. Note that purified recombinant human GARS was analyzed in a similar manner. Optical densities of bands on Western blots were determined using ImageJ software (http://rsb.info.nih.gov/ij/), with background densities subtracted from each measurement. Bandintensity ratios were determined by dividing the optical densities of GARS-specific bands by the optical densities of corresponding actinspecific bands.

Custom rabbit anti-GARS antibodies were generated by Zymed Laboratories using peptides corresponding to four regions of the human GARS protein (amino acid positions 18-31, 302-313, 633-646, and 673-685 in GenBank NP_002038). Peptide-blocking controls were performed using an $\sim 250$-fold excess of the antigenic peptide (Zymed Laboratories).

Construction of GARS- and GRS1-containing expression vectors. The human GARS open reading frame was PCR amplified using a full-length cDNA clone (IMAGE 4109682; GenBank accession number BC007755) as a template. The PCR primers contained flanking NdeI (forward) and XhoI (reverse) restriction sites to allow cloning into the pET-21a(+) expression vector (Novagen) in frame with a C-terminal 6XHis tag. Alternatively, the PCR primers contained flanking PstI (forward) and SmaI (reverse) restriction sites to allow cloning into the pEGFP-N2 expression vector (BD Biosciences, Palo Alto, CA) in frame with a C-terminal enhanced green fluorescent protein (EGFP) tag. The Saccharomyces cerevisiae GRS1 locus (including the open reading frame and $620 \mathrm{bp}$ of upstream sequence) was PCR amplified directly from yeast genomic DNA. The PCR primers contained flanking NotI restriction sites to allow cloning into the NotI site of the yeast vector pRS315 (Sikorski and Hieter, 1989). After suitable restriction enzyme digestion, the PCR-amplified GARS- or GRS1-containing fragments were cloned into the appropriate vector using standard methods. PCR analyses of the resulting bacterial colonies were used to confirm the presence of a GARS- or GRS1containing insert. Subsequent site-directed mutagenesis was performed using the QuikChange site-directed mutagenesis kit (Stratagene) and appropriate mutation-bearing oligonucleotides. An XhoI fragment of the resulting vectors was shuttled into pYES2 (Invitrogen) for overexpression in yeast. All constructs were sequenced to confirm the presence of the designed mutation and the absence of PCR-induced errors.

Yeast growth assays. The yeast strain RJT3/II-1 [his $3 \Delta 200$, leu $2 \Delta 1$, ura3-52, grs 1::HIS3; maintained by a GRS1-containing centromere vector bearing URA3 (Turner et al., 2000)] is devoid of an endogenous GRS1 locus, which was deleted via a recombination with a HIS3-containing cassette containing sequences that flank GRS1 in the yeast genome. This strain was transformed with pRS315 constructs containing wild-type or mutant GRS1 (described above) or no insert. Briefly, lithium acetate transformations (Green et al., 1999) were performed at $30^{\circ} \mathrm{C}$ using $200 \mathrm{ng}$ of plasmid DNA. Transformation reactions were plated on yeast medium lacking uracil and leucine, and the resulting colonies were tested by PCR using primers specific to GRS1 and pRS315. For each transformation, four colonies found to carry the appropriate construct were selected for additional analysis. Each colony was then inoculated into $2 \mathrm{ml}$ of yeast medium lacking uracil and leucine and incubated shaking at $30^{\circ} \mathrm{C}$ for $18 \mathrm{~h}$. Culture dilutions $(1: 1,1: 5,1: 10$, and $1: 125)$ were generated in yeast extract, peptone, and dextrose (YPD) medium (Qbiogene, Irvine, CA), spotted on growth medium containing $0.1 \% 5$-fluoroorotic acid (5-FOA; Teknova, Hollister, CA), and incubated at $30^{\circ} \mathrm{C}$ for $2 \mathrm{~d}$. Growth was assessed by visual inspection. To generate growth curves, surviving yeast colonies were inoculated in selective medium and incubated shaking overnight at $30^{\circ} \mathrm{C}$. Dilutions $(1: 25)$ were then generated in YPD medium, 
A

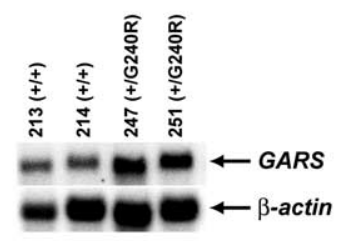

B

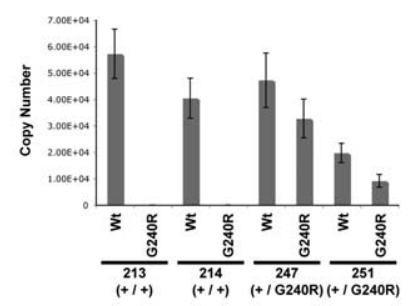

E
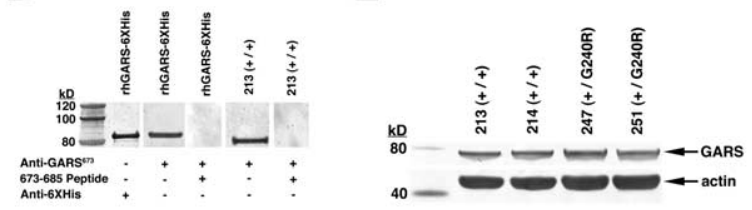

C

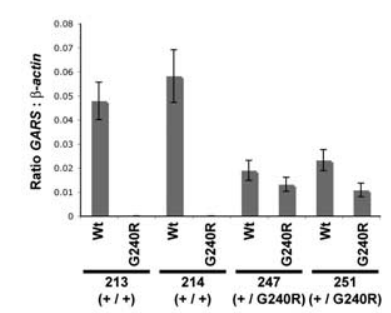

$\mathbf{F}$

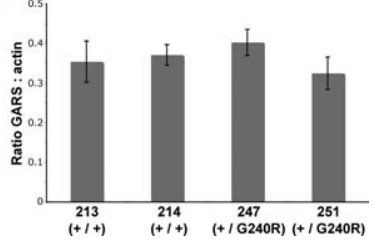

Figure 1. Expression of mutant GARS. A, Total RNA was extracted from lymphoblastoid cell lines derived from unaffected noncarriers $(+/+)$ and CMT2D-affected carriers of the G240R GARS mutation ( $+/ G 240 R)$ and subjected to Northern blot analysis. Blots were hybridized with a GARS- or $\beta$-actin-specific probe, as indicated. $B$, Real-time PCR was performed using reversetranscribed RNA from the cell lines described in $A$ and allele-specific primers. Copy number of the wild-type (Wt) and G240R alleles was measured by including known concentrations of control plasmids containing wild-type or G240R GARS mRNA. C, Real-time PCR studies were performed similarly to those in $\boldsymbol{B}$, except that primers specific for the $\beta$-actin gene were included to assess the efficiency of the reverse-transcription reactions. The ratio of GARS mRNA to $\beta$-actin mRNA was then calculated. $\boldsymbol{D}$, rhGARS- 6 XHis was subjected to Western blot analysis using an anti-GARS ${ }^{673}$ or anti-6XHis antibody, as indicated. In two cases, the hybridization was performed in the presence of excess 673-685 peptide. Similar analyses were performed with a total protein lysate from a lymphoblastoid cell line of an unaffected noncarrier $(+/+)$ using anti-GARS ${ }^{673}$. $E$, Western blot analyses were performed with total protein lysates of lymphoblastoid cell lines from unaffected noncarriers $(+/+)$ and CMT2D-affected carriers of the G240R GARS mutation (+/G240R) using an anti-GARS ${ }^{673}$ or anti-actin antibody, as indicated. $\boldsymbol{F}$, The ratio of GARS to actin protein levels was determined by dividing the optical density of each GARS-specific Western blot band with that of the corresponding actinspecific band (e.g., as in $\boldsymbol{E}$ ) using four independent Western blots. Error bars indicate SEM.

with the new cultures incubated shaking at $30^{\circ} \mathrm{C}$. The $\mathrm{OD}_{660}$ of $1 \mathrm{ml}$ aliquots was then measured at 0, 2, 3, 4, and 5 h using a Beckman DU 650 spectrophotometer (Beckman Coulter, Fullerton, CA). The percentage increase in growth was determined by dividing each of the four measured $\mathrm{OD}_{660}$ values by the initial $\mathrm{OD}_{660}$ value. Curves were generated using the average of the data obtained with the four separate PCR-positive colonies. Similar studies on strains carrying two copies of GRS1 were performed on growth medium containing glycerol or galactose for detecting mitochondrial mutations and dominant-negative effects, respectively.

Cell culture and differentiation. 293T cells, COS7 cells, and the mouse motor neuron cell line MN-1 (Salazar-Grueso et al., 1991) were cultured in DMEM growth medium (Invitrogen) containing 10\% fetal bovine serum, $50 \mathrm{U} / \mathrm{ml}$ penicillin, $50 \mu \mathrm{g} / \mathrm{ml}$ streptomycin, and $2 \mathrm{~mm}$ L-glutamine. The human neuroblastoma cell line SH-SY5Y (Biedler et al., 1978; Ross et al., 1983) was cultured in DMEM/F-12 medium (Invitrogen) containing $10 \%$ fetal bovine serum, $50 \mathrm{~mm}$ HEPES, $50 \mathrm{U} / \mathrm{ml}$ penicillin, $50 \mu \mathrm{g} / \mathrm{ml}$ streptomycin, and $2 \mathrm{~mm}$ L-glutamine. All cells were grown at $37^{\circ} \mathrm{C}$ in $5 \% \mathrm{CO}_{2}$. For cell differentiation, transfection studies, and antibody staining, cultured cells were counted with a hemacytometer, and $\sim 8.3 \times 10^{5}$ cells were placed into each well of a two-well culture slide (BD Biosciences Discovery Labware, Bedford, MA) pretreated with $5 \mu \mathrm{g} / \mathrm{cm}^{2}$ entactin-collagen IV-laminin attachment matrix (Millipore). MN-1 cells were differentiated by the addition of 2 nм glial cell linederived neurotrophic factor (GDNF) and 833 pM GDNF receptor $\alpha-1$ (GFR $\alpha$-1; both from R\&D Systems, Minneapolis, MN) to the culture medium and incubation for $48 \mathrm{~h}$ (Paratcha et al., 2001). SH-SY5Y cells were differentiated by the addition of $10 \mu \mathrm{M}$ all-trans retinoic acid (Sigma) to the culture medium and incubation for $6 \mathrm{~d}$, with the medium changed every $48 \mathrm{~h}$ (Sharma et al., 1999).

Transfection studies. pEGFP-N2 constructs encoding wild-type or mutant GARS tagged with EGFP (described above) or no insert were transfected into cells using the Lipofectamine 2000 reagent (Invitrogen) according to the instructions of the manufacturer. For each reaction, $20 \mu \mathrm{l}$ of Lipofectamine 2000 and $500 \mu \mathrm{l}$ of OptiMEM I minimal growth medium (Invitrogen) were combined and incubated at room temperature for $10 \mathrm{~min}$. Purified plasmid DNA $(2 \mu \mathrm{g})$ or the equivalent volume of water (in the case of negative controls) was diluted in $500 \mu \mathrm{l}$ of OptiMEM I, combined with the above Lipofectamine-OptiMEM I mixture, and incubated at room temperature for $20 \mathrm{~min}$. The entire sample was then added to a single well of a two-well culture slide containing the desired cell line (see above). After a $4 \mathrm{~h}$ incubation at $37^{\circ} \mathrm{C}$, the medium was aspirated, the slides were washed with $1 \times$ PBS, and normal growth medium was added. After a $24 \mathrm{~h}$ incubation at $37^{\circ} \mathrm{C}$, cells were washed with $1 \times$ PBS and then fixed in $4 \%$ paraformaldehyde in $1 \times$ PBS at room temperature for $30 \mathrm{~min}$. Finally, the slides were washed three times in $1 \times$ PBS and coated with ProLong antifade reagent (Invitrogen, Eugene, OR). To visualize mitochondria in COS7 cells, cells were transfected with the pDsRed2-Mito vector (BD Biosciences) as described above.

Antibody staining. Human SH-SY5Y cells were cultured at $37^{\circ} \mathrm{C}$ for $6 \mathrm{~d}$ in differentiation medium (see above) using two-well culture slides (BD Biosciences Discovery Labware). Cells were fixed in $4 \%$ paraformaldehyde, permeabilized in $0.2 \%$ Triton X-100 in $1 \times$ PBS for $5 \mathrm{~min}$, washed in $1 \times \mathrm{PBS}$, and incubated in blocking solution containing $10 \%$ normal goat serum $/ 1 \times \mathrm{PBS} / 0.05 \%$ sodium azide (Zymed Laboratories) for $30 \mathrm{~min}$. Cells were then incubated in $10 \mu \mathrm{g} / \mathrm{ml}$ anti-GARS ${ }^{673}$ in blocking solution for $60 \mathrm{~min}$. After three washes in $1 \times$ $\mathrm{PBS}$, cells were incubated in goat anti-rabbit IgG secondary antibody conjugated with FITC (Zymed Laboratories) for $60 \mathrm{~min}$. After three washes in $1 \times$ PBS, cells were incubated in $300 \mathrm{~nm}$ 4',6-diamidino-2-phenylindole dihydrochloride (DAPI; Invitrogen) in $1 \times$ PBS for $5 \mathrm{~min}$. Cells were washed three times in $1 \times$ PBS, the well apparatus was removed, and slides were coated with ProLong antifade reagent (Invitrogen). All incubation and wash steps were performed at room temperature. To detect other cellular structures, cells were costained with mouse anti-bovine $\alpha$-tubulin (1:50; Invitrogen), mouse anti-human survival motor neuron (SMN) (1:100; BD Biosciences Pharmingen, San Jose, CA), mouse anti-nucleolar antigen (1:30; GeneTex, San Antonio, TX), or mouse anti-OxPhos complex IV (1:100; Invitrogen) followed by a goat anti-mouse IgG secondary antibody conjugated with tetramethylrhodamine isothiocyanate (Zymed Laboratories), as described above.

Formalin-fixed, paraffin-embedded tissue sections were subjected to two $10 \mathrm{~min}$ incubations in xylene, after which the slides were rinsed in a series of 2 min ethanol washes $(100,95$, and $80 \%$ ) followed by a 5 min rinse in deionized water. Slides were then placed in $50 \mathrm{ml}$ conical tubes containing $37.5 \mathrm{ml}$ of target retrieval solution (DakoCytomation, Carpinteria, CA), incubated in a $95^{\circ} \mathrm{C}$ water bath for $30 \mathrm{~min}$, cooled at room temperature for $20 \mathrm{~min}$, and rinsed three times in $1 \times$ PBS for $5 \mathrm{~min}$ each. The resulting tissue sections were then stained with anti-GARS ${ }^{673}$ and DAPI, as described above. To visualize axons, sections were stained with mouse anti-human phosphorylated neurofilament (1:320; DakoCytomation).

Fluorescence microscopy and image collection. Microscopic images were obtained using either a Zeiss model LSM 510 confocal fluorescence microscope (Zeiss, Oberkochen, Germany) or a Zeiss Axiovert 200M fluorescence microscope (Zeiss) equipped with an ORCA-ER digital camera (Hamamatsu Photonics, Hamamatsu, Japan) using Openlab image software version 4.0.2 (Improvision, Lexington, MA). 


\section{Results}

\section{The G240R GARS mutation does not impair transcription} or translation

We analyzed lymphoblastoid cell lines from CMT2D patients and appropriate controls by Northern blot analyses to assess the relative levels of GARS mRNA. G240R heterozygotes (+/G240R) and noncarriers $(+/+)$ express appropriately spliced GARS mRNA $(\sim 2.7 \mathrm{~kb})$ at similar levels (Fig. $1 A)$. To ensure that mutant RNA is expressed and stable, we performed reverse transcription-PCR and DNA sequencing with the RNA samples. This revealed the presence of both wild-type and G240R GARS alleles in the mRNA population of G240R heterozygotes (data not shown), indicating that the G240R mutation does not reflect a null allele. In addition, we performed reverse transcription and allele-specific real-time PCR on the above samples to quantify the levels of each GARS allele (Fig. $1 B, C$ ). This revealed that both alleles are expressed, albeit with the G240R allele showing slightly lower expression than the wild-type allele. Thus, the G240R mutation does not cause a severe reduction in GARS mRNA levels in lymphoblastoid cell lines.

We also performed Western blot analyses using a rabbit antipeptide antibody raised against residues 673-685 (antiGARS $^{673}$ ) of the human GARS protein. When analyzing recombinant human GARS tagged with 6XHis (rhGARS-6XHis), antiGARS $^{673}$ detects a similarly sized protein (slightly $>80 \mathrm{kDa}$ ) as an anti-6XHis antibody (Fig. 1D). Factoring in the size of the 6XHis tag, these data are consistent with the previously predicted molecular weight of human GARS [between 77 and $80 \mathrm{kDa}$ (Targoff, 1990; Hirakata et al., 1992; Williams et al., 1995)]. The 673-685 peptide abolishes detection of this band with anti-GARS ${ }^{673}$.

Western blot analysis of a total protein sample from a noncarrier $(+/+)$ lymphoblastoid cell line with anti-GARS ${ }^{673}$ reveals a protein $\sim 80 \mathrm{kDa}$ in size (Fig. $1 D$ ); once again, the $673-685$ peptide abolishes detection of this band. Interestingly, a smaller band ( $\sim 60 \mathrm{kDa}$ in size) is also detected by anti-GARS ${ }^{673}$ in approximately half of the analyses, and addition of the 673-685 peptide also abolishes the detection of this band (data not shown). A GARS variant with this molecular weight has not been described (although the assessment of GARS size in lymphoblastoid cell lines has not been reported previously). This smaller protein may represent a novel GARS variant or a GARS-related protein with an epitope similar to the 673-685 peptide. Visual inspection of Western blots (Fig. $1 E$ ) and quantitative analysis of the optical densities of GARS- and actin-specific bands (Fig. $1 F$ ) suggest that lymphoblastoid cell lines from G240R heterozygotes (+/G240R) and noncarriers $(+/+)$ express similar levels of the GARS protein. Thus, the G240R mutation does not lead to a severe reduction in GARS protein levels in lymphoblastoid cell lines.

Most GARS mutations modeled in yeast cause growth defects We studied the effects of GARS mutations modeled in GRS1. Specifically, GRS1 was mutagenized to create analogous versions of five GARS mutations identified in CMT2D and dSMA-V patients (Table 1). For each of these mutations, the amino acid affected by the human GARS mutation is identical in GRS1. The various forms of GRS1 were generated in a yeast centromere vector bearing HIS3; each in turn was transfected into the yeast strain RJT3/II-1 (Turner et al., 2000). In this strain, the endogenous GRS1 locus is deleted; however, because GRS1 is an essential gene, this strain carries a yeast centromere vector-bearing wildtype GRS1 and URA3 to maintain viability.

Haploid yeast carrying an inactive form of GRS1 would not be expected to grow, because GRS1 is an essential gene (Turner et al.,
Table 1. Human GARS mutations modeled in the yeast ortholog GRS1

\begin{tabular}{ll}
\hline Human GARS mutation $^{a}$ & Yeast GRS1 mutation $^{b}$ \\
\hline E71G & E21G \\
L129P & L79P \\
G240R & G223R \\
H418R & H402R \\
G526R & G512R
\end{tabular}

${ }^{a}$ Amino acid coordinates correspond to the sequence in GenBank NP_002038.

${ }^{b}$ Amino acid coordinates correspond to the sequence in GenBank NP_009679.

A

B

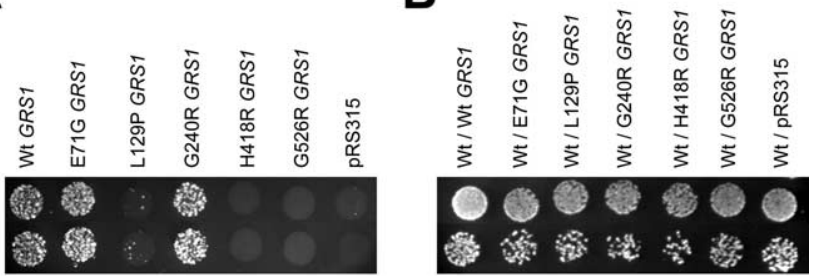

C

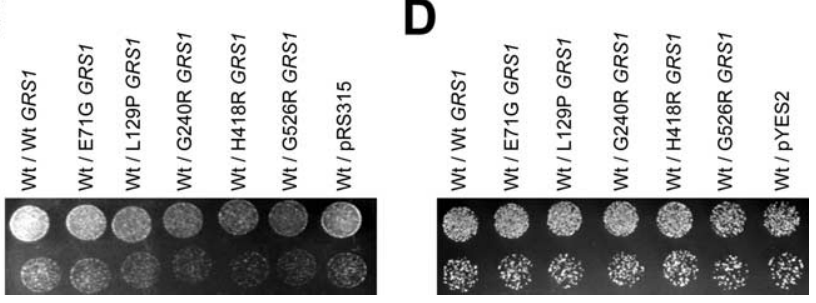

Figure 2. Growth of yeast strains containing wild-type and mutant GRS1.A, Representative cultures of the indicated yeast strains were inoculated and grown on medium containing 5-FOA. Each strain had been transfected previously with a vector containing no insert (pRS315), wildtype (Wt) GRS1, or the indicated mutant form of GRS1 that modeled a human GARS mutation (Table 1). $\boldsymbol{B}$, Representative cultures of the indicated yeast strains were inoculated and grown on medium containing glucose. Each strain contains the wild-type GRS1-bearing maintenance vector (see Materials and Methods for details) as well as a transformed vector expressing the indicated GRS1 allele from the endogenous promoter. The Wt/pRS315 strain contained a transformed vector without an insert. $\boldsymbol{C}$, Similar experiment to that in $\boldsymbol{B}$, except that strains were grown on medium containing glycerol. $\boldsymbol{D}$, Representative cultures of the indicated strains were inoculated and grown on medium containing galactose; each strain contained two copies of $G R S 1$, one wild-type copy expressed from the endogenous promoter and one expressing the indicated allele from the GAL1 promoter.

2000). To test the viability of yeast strains bearing GRS1 mutations, we used 5-FOA treatment (Boeke et al., 1984) to eliminate the URA3-containing vector (along with wild-type GRS1). Subsequently, each strain was examined for growth and viability. Three of the modeled GARS mutations (corresponding to human mutations L129P, H418R, and G526R) show substantial reductions in yeast viability compared with wild-type GRS1 (Fig. 2A). In contrast, two modeled GARS mutations (corresponding to human mutations E71G and G240R) do not appear to have a major effect on yeast viability. Because GRS1 (as well as GARS) charges tRNA in both the cytoplasm and mitochondria (Shiba et al., 1994; Turner et al., 2000), we tested the ability of strains surviving 5-FOA treatment to grow on media containing glycerol, which forces the use of mitochondria for ATP production. This analysis did not reveal any growth defects associated with GRS1 mutations (data not shown).

GARS and GRS1 are predicted to exist as homodimers in vivo. Thus, it is possible that the presence of a mutant subunit in the homodimer ablates enzyme function by a dominant-negative mechanism. To study this, we examined the growth of yeast strains carrying two copies of the GRS1 gene. First, we analyzed the growth of strains carrying two GRS1 alleles, both expressed 

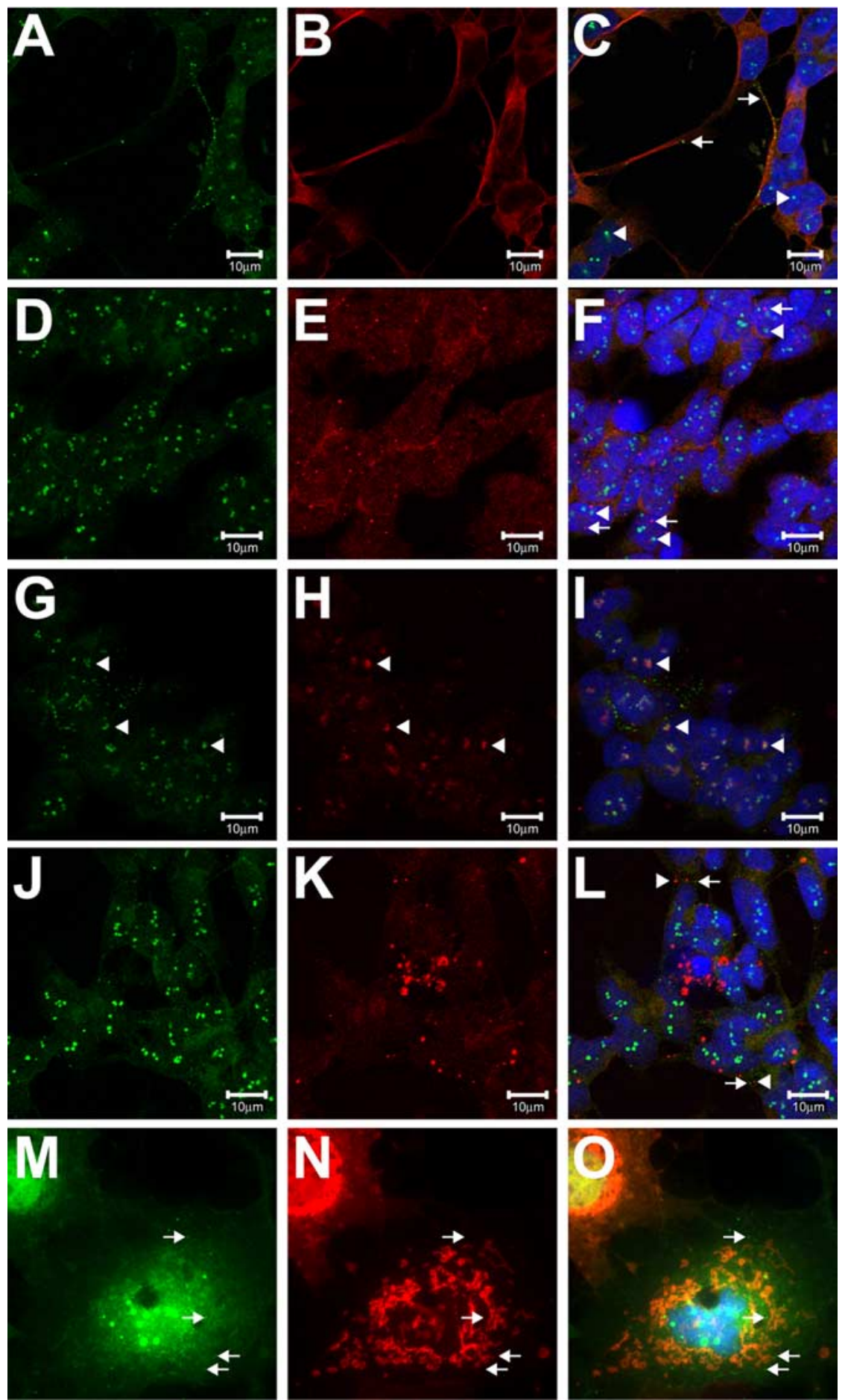

Figure 3. Expression of endogenous GARS in cultured cells. $\boldsymbol{A}, \boldsymbol{B}, \boldsymbol{D}, \boldsymbol{E}, \boldsymbol{G}, \boldsymbol{H}, \boldsymbol{J}, \boldsymbol{K}$, Differentiated human SH-SY5Y cells were stained with anti-GARS ${ }^{673}(\boldsymbol{A}, \boldsymbol{D}, \boldsymbol{G}, \boldsymbol{J})$, anti- $\alpha$-tubulin $(\boldsymbol{B})$, anti-SMN $(\boldsymbol{E})$, anti-nucleolar antigen $(\boldsymbol{H})$, anti-0xPhos complex IV $(\boldsymbol{K})$, and DAPI and examined by confocal fluorescence microscopy. $C, \boldsymbol{F}, \boldsymbol{I}, \boldsymbol{L}$, Merged images depict large GARS-associated globules in the nuclei ( $\boldsymbol{C}$, arrowheads) and granules in the neurite projections ( $\boldsymbol{C}$, arrows); distinct and separate localization of SMN gems $(\boldsymbol{F}$, arrows) and nuclear GARS-associated globules ( $\boldsymbol{F}$, arrowheads); multiple sites of colocalized GARS-associated globules and nucleoli in the nucleus ( $\boldsymbol{I}$, arrowheads); and distinct cytoplasmic GARS-associated granules ( $\boldsymbol{L}$, arrows) in the absence of mitochondrial staining ( $\boldsymbol{L}$, arrowheads). $\boldsymbol{M - 0}, \operatorname{COS7}$ cells were stained with anti-GARS ${ }^{673}$ and DAPI in the absence $(\boldsymbol{M})$ or presence $(\boldsymbol{N})$ of the transfected pDsRed2-Mito vector. The merged image $(\boldsymbol{0})$ shows GARS-associated granules (arrows) distinct from the labeled mitochondria.

with the endogenous promoter and cultured in media containing either glucose (Fig. $2 B$ ) or glycerol (Fig. 2C). In addition, we performed allele-specific real-time PCR to ensure that both GRS1 alleles were expressed at similar levels. These experiments were successfully performed for three of the mutant GRS1 forms (corresponding to human mutations G240R, H418R, and G526R); in each case, the mutant GRS1 allele was found to be expressed at a similar (or higher) level as the wild-type allele (data not shown). Next, we analyzed the growth of strains carrying one GRS1 allele expressed with the endogenous promoter and a second allele overexpressed with the GAL1 promoter by culturing in galactose-containing media (Fig. 2D). These experiments did not reveal mutation-associated impairment of yeast cell growth, indicating that GARS mutations modeled in GRS1 do not cause a dominant-negative mechanism in yeast cells. Growth curve studies performed with all of the same strains were consistent with this conclusion (data not shown).

\section{Endogenous GARS is associated with granules in cultured neuron cells}

The cellular and tissue distribution of GARS has not been reported. To begin exploring the cellular localization of the enzyme, we studied endogenous GARS in the human neuroblastoma cell line $\mathrm{SH}$ SY5Y, which can be differentiated with retinoic acid to form long neurite projections (Sharma et al., 1999). Staining differentiated SH-SY5Y cells with antiGARS $^{673}$, anti- $\alpha$-tubulin, and DAPI revealed the presence of large GARSassociated granules (or globules) in the nucleus and smaller GARS-associated granules in the cell body and neurite projections (Fig. 3A-C).

$S M N$ is mutated in juvenile-onset spinal muscular atrophy and encodes an RNA-binding protein that localizes to gems [Gemini of the coiled bodies (Liu and Dreyfuss, 1996)] in cell nuclei. We stained differentiated SH-SY5Y cells with anti-GARS ${ }^{673}$, anti-SMN, and DAPI, and found no evidence for colocalization of the GARS and SMN proteins (Fig. 3D-F). Methionine-tRNA synthetase (MARS) was previously localized to the nucleolus of five cell lines and implicated in ribosomal RNA synthesis (Ko et al., 2000). We stained differentiated SH-SY5Y cells with anti-GARS ${ }^{673}$, anti-nucleolar antigen, and DAPI and found that GARS also localizes to the nucleolus (Fig. 3G-I). These novel findings were replicated in non-neuronal cells (293T and COS7) (data not shown), indicating that the cellular localizations of these proteins are not unique to neurons.

Because GARS is known to function in both the cytoplasm and mitochondria (Shiba et al., 1994; Turner et al., 2000), we tested whether the GARS-associated granules represent mitochondria. Two cell populations were prepared for this: (1) differentiated SH-SY5Y cells stained with anti-OxPhos 
complex IV; and (2) COS7 cells transfected with a construct expressing a mitochondrial-targeting sequence tagged with Discosoma sp. red fluorescent protein (DsRed). Both cell populations were then stained with anti-GARS ${ }^{673}$ and DAPI, in both cases revealing GARS-associated granules distinct from the labeled mitochondria (Fig. 3J-O). Thus, the GARSassociated granules do not appear to represent mitochondria. In similar studies involving differentiated SH-SY5Y cells stained with anti-Golgin-97, anti-GARS ${ }^{673}$, and DAPI, we found that the GARSassociated granules do not appear to represent the Golgi apparatus (data not shown).

Because a subset of aminoacyl-tRNA synthetases reside in multisynthetase complexes (MSCs) (thought to raise the efficiency of tRNA charging) (Han et al., 2003), we also tested whether the GARSassociated granules represent such complexes. Specifically, we examined whether GARS-associated granules colocalize with tRNA synthetases shown previously to reside within [MARS and glutaminyl-tRNA synthetase (QARS)] or outside of [seryltRNA synthetase (SARS)] such multisynthetase complexes (Han et al., 2003). This involved transfecting SH-SY5Y cells with vectors expressing MARS, QARS, or SARS tagged with DsRed and staining the cells with anti-GARS ${ }^{673}$, followed by confocal microscopic analysis. In no case did we find evidence for the colocalization of the GARSassociated granules and other aminoacyltRNA synthetases (data not shown).

\section{GARS localizes to peripheral nerve axons in vivo}

We also investigated the location of the GARS protein in human neuronal tissue. Human thoracic spinal cord and sural nerve tissue sections (derived from presumably normal adult cadavers) were stained with anti-human neurofilament, anti-GARS ${ }^{673}$, and DAPI. These studies revealed strong anti-GARS ${ }^{673}$ staining in both the ventral (Fig. $4 A-C$ ) and dorsal (Fig. 5A-C) horns of the spinal cord. In addition, axons of the ventral (Fig. 4G-I) and dorsal (Fig. 5D-F) roots showed strong anti-GARS ${ }^{673}$ staining, indicating that GARS is present outside of the neuronal cell body. Closer examination revealed that GARS is associated with distinct granules in the axons of the ventral horn (Fig. 4D-F), dorsal horn (data not shown), ventral root (Fig. $4 J-L$ ), dorsal root (Fig. 5G-I), and sural nerve (Fig. 5J-L). Finally, anti-GARS ${ }^{673}$ staining was also seen in the nuclei of cells in all of the above structures (Fig. 5I,L, and data not shown).

\section{Most mutant forms of GARS are mislocalized in cultured} motor neuron cells

The mouse motor neuron cell line MN-1 (Salazar-Grueso et al., 1991) can be differentiated with GDNF and GFR $\alpha$-1 (Paratcha et al., 2001). During differentiation, long neurite projections form, providing an in vitro model of nerve cell axons. To determine the effect of GARS mutations on cellular localization, we transfected MN-1 cells with expression vectors containing wild-type or mutant GARS tagged with EGFP; the resulting cell populations were then differentiated with GDNF and GFR $\alpha$-1 and examined by fluorescence microscopy.

Wild-type GARS-EGFP associates with granules in both the cell body and neurite projections of $42 \%$ of EGFP-positive MN-1 cells (Fig. 6A-D,G). In contrast, L129P GARS-EGFP (Fig. 6E), G240R GARS-EGFP (Fig. 6F), and EGFP alone (Fig. 6G) never associate with such granules, whereas E71G GARS-EGFP, H418R GARS-EGFP, and G526R GARS-EGFP associate with these granules in 36,1 , and $20 \%$ of EGFP-positive cells, respectively 

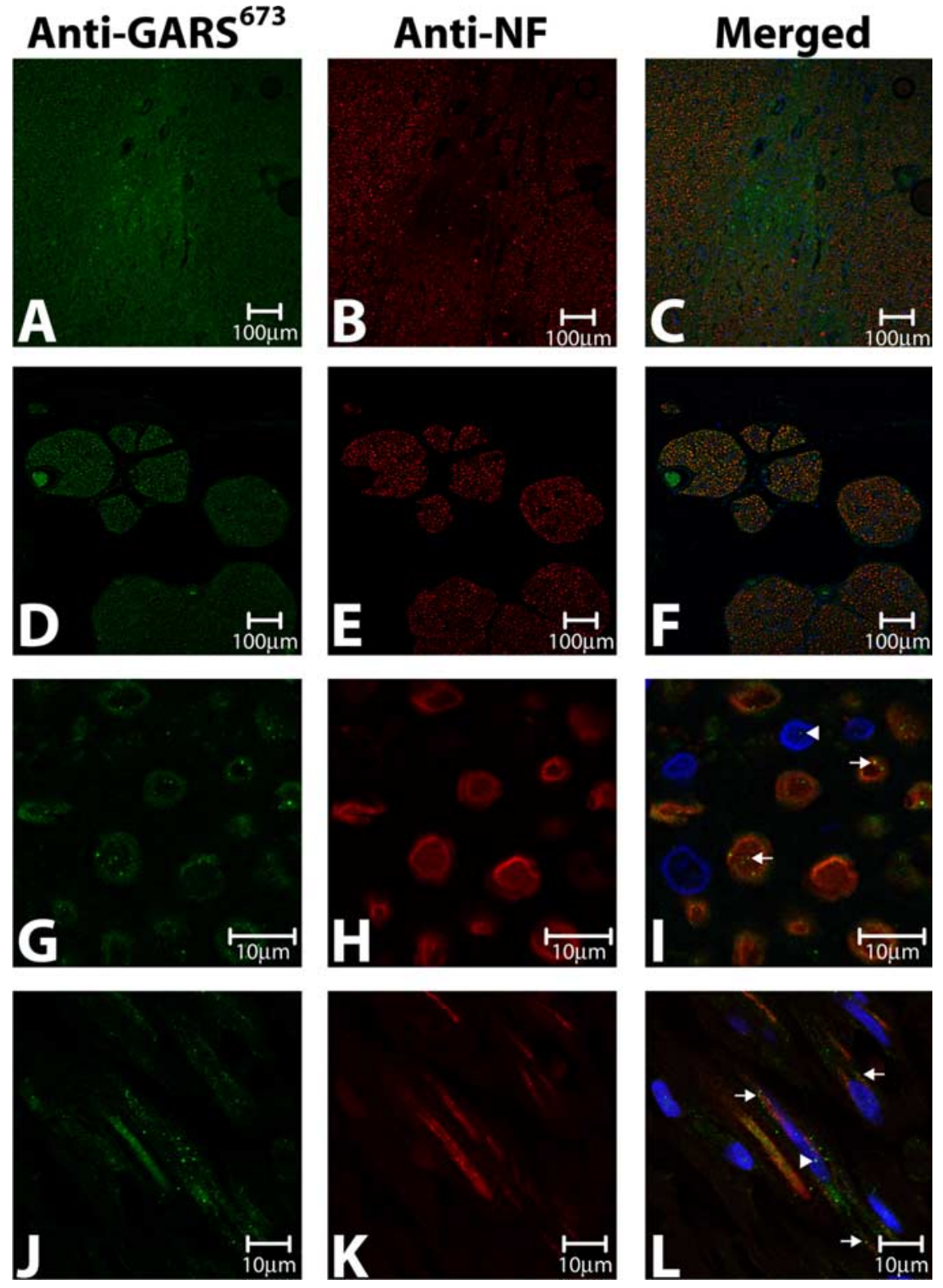

Figure 5. Localization of GARS in the dorsal horn, dorsal root, and sural nerve. Human thoracic spinal cord $(\boldsymbol{A}-\boldsymbol{I})$ and sural nerve $(\boldsymbol{J}-\boldsymbol{L})$ sections were stained with anti-GARS ${ }^{673}(\boldsymbol{A}, \boldsymbol{D}, \boldsymbol{G}, \boldsymbol{J})$, anti-neurofilament (anti-NF; $\boldsymbol{B}, \boldsymbol{E}, \boldsymbol{H}, \boldsymbol{K}$ ), and DAPI and examined by confocal fluorescence microscopy. Merged images show strong GARS staining in the dorsal horn ( $\boldsymbol{C}$, strong GARS staining in dorsal root axons $(\boldsymbol{F})$, and GARS-associated granules in dorsal root ( $\boldsymbol{I}$, arrows) and sural nerve ( $\boldsymbol{L}$, arrows) axons. Note that GARS staining can also be seen in nuclei of the dorsal root and sural nerve ( $I, L$, arrowheads).

(Fig. 6G). Similar results were seen when these constructs were transfected into human endothelial kidney 293T cells (data not shown). Thus, four of the five GARS mutations studied give rise to a markedly different protein localization pattern compared with the wild-type enzyme.

\section{Discussion}

Our previous identification of GARS as the gene mutated in CMT2D and dSMA-V (Antonellis et al., 2003) was somewhat unexpected, because the encoded protein (GARS) is ubiquitously expressed and plays a global role in protein synthesis. Understanding how GARS mutations lead to the highly specific phenotypic features of these peripheral neuropathies (Christodoulou et al., 1995; Ionasescu et al., 1996; Sambuughin et al., 1998; Antonellis et al., 2003; Sivakumar et al., 2005) requires additional insight about the mutant forms of GARS found in affected individuals. Here, we present studies examining the functional consequences of GARS mutations as well as some natural characteristics of GARS that reveal important clues about the molecular pathology of these inherited diseases.

A common pathological mechanism for genetic disorders is a loss of function through altered mRNA or protein levels, although this is unusual for neurodegenerative diseases inherited in an autosomal dominant manner. Based on several studies (Fig. 1), there does not appear to be evidence for severely altered transcription, translation, or protein stability associated with the G240R GARS mutation (the one for which lymphoblastoid cell lines are available). However, our studies did not examine GARS expression in neurons or the effects of other GARS mutations. Such studies should be performed before definitively concluding that CMT2D and dSMA-V do not arise from altered levels of GARS.

Modeling human GARS mutations in the yeast ortholog (GRS1) revealed that several mutations (L129P, H418R, and G526R) do not complement removal of wild-type GRS1, whereas others (E71G and G240R) appear to do so. Interestingly, L129P, H418R, and G526R are associated only with dSMA-V, whereas E71G is associated with either CMT2D or dSMA-V, and G240R is only associated with CMT2D. Thus, mutations showing a loss of function in yeast are most closely associated with dSMA-V (but not CMT2D). In light of the phenotypic similarity of CMT2D and dSMA-V, one explanation is that all mutations cause a loss of function, but that only those associated with dSMA-V are severe enough to detect a phenotype in yeast. Interestingly, two GARS mutations have been associated with both CMT2D and dSMA-V (Sambuughin et al., 1998; Antonellis et al., 2003; Del Bo et al., 2006). Thus, if the above speculation is correct, there are perhaps modifiers of GARS mutations that promote development of either the CMT2D or $\mathrm{dSMA}-\mathrm{V}$ phenotype. Identifying such modifiers might aid diagnosis and understanding the molecular basis of these diseases.

Our yeast studies did not detect a dominant-negative effect of GRS1 mutations. This is surprising because GARS is predicted to exist as a homodimer (Freist et al., 1996), and analogous yeastmodeling studies of disease-causing mutations in the YARS gene indicated such a dominant-negative effect in another variant of CMT (Jordanova et al., 2006). Perhaps yeast assays are not sensitive enough to detect a dominant-negative effect of GARS mutations. However, there are a number of possibilities that could explain the molecular pathology of GARS mutations that do not involve a dominant-negative effect on the homodimer. First, the 

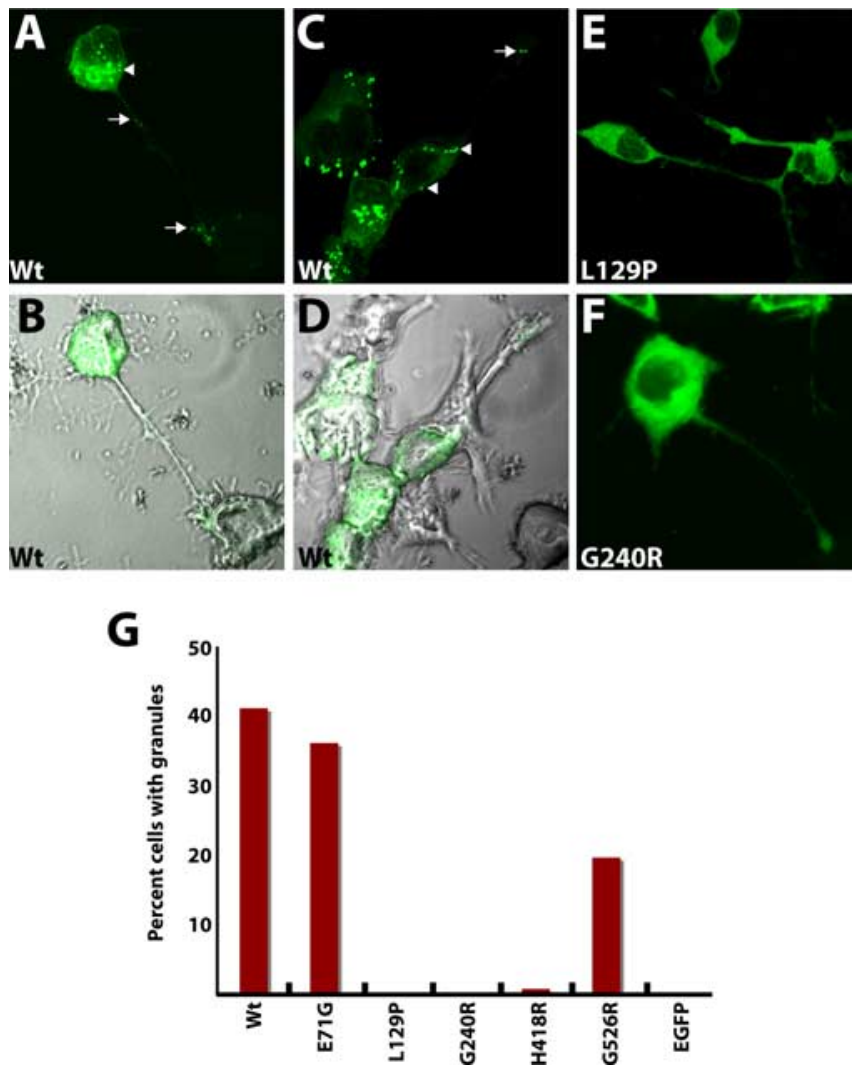

Figure 6. Expression of wild-type and mutant GARS-EGFP in mouse motor neuron cells. $A$, $\boldsymbol{C}, \boldsymbol{E}, \boldsymbol{F}$, Differentiated MN-1 cells expressing wild-type (Wt) GARS-EGFP $(\boldsymbol{A}, \mathrm{C}), \mathrm{L} 129 \mathrm{P}$ GARSEGFP $(\boldsymbol{E})$, or G240R GARS-EGFP $(\boldsymbol{F})$ were examined by fluorescence microscopy. Wild-type GARS-EGFP-associated granules within the cell body and neurite projections are indicated by arrowheads and arrows, respectively. $\boldsymbol{B}, \boldsymbol{D}$, Merged images from fluorescence and differential interference contrast microscopy reveal cell morphology and neurite-projection paths of cells expressing wild-type GARS-EGFP. G, The percentage of EGFP-positive cells containing GARSassociated granules was determined after transfection with constructs expressing wild-type GARS-EGFP, the indicated mutant forms of GARS-EGFP, or EGFP alone. Five replicate experiments consistently gave the observed results.

peripheral neuropathy seen in dSMA-V and CMT2D may be caused by haploinsufficiency of GARS (i.e., a 50\% reduction in protein function). This seems less likely because haploinsufficiency is rarely associated with autosomal dominant neurodegenerative diseases. Nonetheless, it is intriguing to consider that very long axons may be particularly susceptible to a $50 \%$ reduction in the activity of certain enzymes that are required locally. Second, GARS mutations may cause a transdominant negative effect that alters another aspect of enzyme function. For example, they may impair the function of a multienzyme complex (such as the GARS-associated granules). Finally, the observed loss of function described here for GARS mutations may not be the true cause of the peripheral neuropathy (see below).

Protein-localization studies involving a mouse neuronal cell line provided insight about the aggregation and cellular distribution of wild-type and mutant forms of GARS. Specifically, these studies showed that wild-type GARS is associated with granules in the cell body and neurite projections, whereas the majority of the mutant forms of GARS are not. Two interesting considerations arise from these findings. First, the altered cellular distribution of mutant GARS may promote aberrant protein aggregation, possibly in a neuron-specific manner. Thus, whereas our in vitro cell-culture assays did not detect aggregation of mutant proteins, GARS aggregation may occur in vivo, leading to impaired
Table 2. Summary of functional studies of GARS mutations

\begin{tabular}{llll}
\hline GARS mutation & Human phenotype & Yeast viability ${ }^{a}$ & Granule formation $^{b}$ \\
\hline Wild type & None & + & Yes \\
E71G & CMT2D or dSMA-V & + & Yes \\
L129P & dSMA-V & $+/-$ & Impaired \\
G240R & CMT2D & + & Impaired \\
H418R & dSMA-V & - & Impaired \\
G526R & dSMA-V & - & Yes $^{c}$ \\
\hline
\end{tabular}

${ }^{a}+$, Normal viability; + /-, severely impaired growth, but not lethal; - , lethal.

${ }^{b}$ Granule formation as detected in transfected MN-1 (Fig. 6) and 293T cells.

'Granule formation present but reduced.

axonal transport (or function) and the pathological axonopathy. The identification of GARS-associated granules within axons is consistent with this notion. Such a gain-of-function mechanism is thought to underlie many neurodegenerative diseases (Wood et al., 2003) and needs to be fully explored before any definitive conclusions are made about the molecular pathology of CMT2D and dSMA-V.

However, this redistribution of the mutant protein is consistent with a loss-of-function mechanism. Thus, between the yeast studies and the examination of GARS-associated granules in a neuronal cell line, all GARS mutations except E71G show evidence of a loss-of-function mechanism (Table 2). E71G may cause a loss of GARS function that is not detectable as an effect on yeast growth or on protein distribution in cell culture. Two experimental approaches can be envisioned to investigate this further. First, the in vitro biochemical activity of the different mutant forms of GARS could be assayed to assess the tRNA-charging capacity of each. Second, mRNA and protein levels could be studied in cell lines from a patient harboring the E71G mutation to look for impaired transcription or translation; however, no such cell lines are available at the present time.

The identification of GARS-associated cytoplasmic granules and nuclear globules is a novel finding and raises immediate questions about their functional role(s). For proper protein translation in eukaryotic cells, tRNA molecules must be charged in both the cytoplasm and mitochondria. For some tRNA species, a single aminoacyl-tRNA synthetase is responsible for both cytoplasmic and mitochondrial charging, whereas for others, there are separate enzymes. GARS is thought to aminoacylate tRNA Gly molecules in both locations (Shiba et al., 1994; Turner et al., 2000). Furthermore, aminoacyl-tRNA synthetases form cytoplasmic MSCs thought to enhance aminoacylation efficiency and composed of at least eight aminoacyl-tRNA synthetases and the nonenzyme anchor proteins p18, p38, and p48 (Han et al., 2003). Thus, the cytoplasmic GARS-associated granules could potentially represent mitochondria or MSCs. However, the results reported here (Fig. 3) indicate that the GARS-associated granules represent neither mitochondria or MSCs, suggesting that they represent a previously unidentified structure. Our studies also indicate that GARS localizes to the nucleolus in cultured cells. Interestingly, methionyl-tRNA synthetase has been localized to the nucleolus and is predicted to play a role in ribosomal RNA biogenesis (Ko et al., 2000). Determining the role of GARS in the nucleolus and cytoplasmic granules is of interest and will likely be important for understanding dSMA-V and CMT2D pathogenesis.

GARS missense mutations give rise to an axonal peripheral neuropathy. If, indeed, these mutations act via a loss-of-function mechanism, then there must be a unique role for GARS in peripheral nerve axons (in light of the limited phenotype of 
dSMA-V and CMT2D and the ubiquitous requirement of GARS in all cells). We believe that our identification of GARSassociated granules in vivo, which is the first report of a human aminoacyl-tRNA synthetase localizing to axons, provides a clue about such a unique role. Perhaps the GARS-associated granules facilitate local tRNA charging and translation. Indeed, local protein synthesis in axons has been suggested by the identification of translational activity and ribosomes in isolated mammalian axoplasm, tRNAs and aminoacyl-tRNA synthetases in squid axoplasm, and mRNA in the axoplasm of multiple vertebrates (Giuditta et al., 2002). Therefore, GARS may be needed in axons to charge recycled tRNA ${ }^{\text {Gly }}$ molecules and support local protein synthesis, with its absence or dysfunction leading to CMT2D or dSMA-V. Our cell culture studies suggest that GARS mutations can abrogate the association of the GARS protein with granules. This raises the possibility that the proper localization of GARS within granules and the subsequent transport of the granules to the periphery are essential for maintaining axonal health; in essence, this would represent a special requirement for GARS in peripheral nerve cell axons.

The recent finding that mutations in YARS cause dominant intermediate CMT (Jordanova et al., 2006), a peripheral neuropathy with both demyelinating and axonal features, is relevant to the studies reported here. Specifically, the association between peripheral neuropathy and mutations in two genes with an important role in protein synthesis indicates that peripheral nerves are uniquely susceptible to impaired or altered protein synthesis. Furthermore, impaired degradation of a protein essential for assembly of the multiple tRNA synthetase complex [p38/JTV1 (Kim et al., 2002; Corti et al., 2003; Ko et al., 2005)] is associated with neurodegeneration in certain patients with Parkinson's disease, indicating that neurons in general may be more susceptible to protein synthesis defects.

Based on our studies, we propose that the peripheral neuropathy in CMT2D and ASMA-V is caused by impaired GARS enzyme activity, a GARS-localization defect, or a combination of both. Perhaps a fundamental aspect of these diseases is the level of functional GARS present in peripheral nerve axons. Individuals with two wild-type GARS alleles presumably have $100 \%$ functional GARS in the cell bodies of peripheral neurons, with a subpopulation of GARS then transported to the periphery to support axonal tRNA ${ }^{\text {Gly }}$ charging. GARS-mutation carriers likely have decreased GARS activity levels, perhaps falling below some critical (and unique) threshold in the axon that leads to defective axonal translation and axonal damage. Such a hypothesis is consistent with the progressive nature of the disease as well as its peripheral axon-specific pathology. Furthermore, non-neuronal cells, neuron cell bodies, and axons spanning shorter distances may be less sensitive to decreased GARS activity levels. Future studies that further test this hypothesis and examine possible gain-of-function mechanisms should provide more detailed insight into the etiology of CMT2D and dSMA-V as well as the role of tRNA-charging enzymes in maintaining healthy peripheral axons.

\section{References}

Antonellis A, Ellsworth RE, Sambuughin N, Puls I, Abel A, Lee-Lin SQ, Jordanova A, Kremensky I, Christodoulou K, Middleton LT, Sivakumar K, Ionasescu V, Funalot B, Vance JM, Goldfarb LG, Fischbeck KH, Green ED (2003) Glycyl tRNA synthetase mutations in Charcot-Marie-Tooth disease type $2 \mathrm{D}$ and distal spinal muscular atrophy type V. Am J Hum Genet 72:1293-1299.

Biedler JL, Roffler-Tarlov S, Schachner M, Freedman LS (1978) Multiple neurotransmitter synthesis by human neuroblastoma cell lines and clones. Cancer Res 38:3751-3757.

Boeke JD, LaCroute F, Fink GR (1984) A positive selection for mutants lacking orotidine- $5^{\prime}$-phosphate decarboxylase activity in yeast: 5-fluoroorotic acid resistance. Mol Gen Genet 197:345-346.

Christodoulou K, Kyriakides T, Hristova AH, Georgiou DM, Kalaydjieva L, Yshpekova B, Ivanova T, Weber JL, Middleton LT (1995) Mapping of a distal form of spinal muscular atrophy with upper limb predominance to chromosome 7p. Hum Mol Genet 4:1629-1632.

Corti O, Hampe C, Koutnikova H, Darios F, Jacquier S, Prigent A, Robinson JC, Pradier L, Ruberg M, Mirande M, Hirsch E, Rooney T, Fournier A, Brice A (2003) The p38 subunit of the aminoacyl-tRNA synthetase complex is a Parkin substrate: linking protein biosynthesis and neurodegeneration. Hum Mol Genet 12:1427-1437.

Del Bo R, Locatelli F, Corti S, Scarlato M, Ghezzi S, Prelle A, Fagiolari G, Moggio M, Carpo M, Bresolin N, Comi GP (2006) Coexistence of CMT-2D and distal SMA-V phenotypes in an Italian family with a GARS gene mutation. Neurology 66:752-754.

Dyck PJ, Lambert EH (1968) Lower motor and primary sensory neuron diseases with peroneal muscular atrophy. II. Neurologic, genetic, and electrophysiologic findings in various neuronal degenerations. Arch Neurol 18:619-625.

Evgrafov OV, Mersiyanova I, Irobi J, Van Den Bosch L, Dierick I, Leung CL, Schagina O, Verpoorten N, Van Impe K, Fedotov V, Dadali E, AuerGrumbach M, Windpassinger C, Wagner K, Mitrovic Z, Hilton-Jones D, Talbot K, Martin JJ, Vasserman N, Tverskaya S, et al. (2004) Mutant small heat-shock protein 27 causes axonal Charcot-Marie-Tooth disease and distal hereditary motor neuropathy. Nat Genet 36:602-606.

Freist W, Logan DT, Gauss DH (1996) Glycyl-tRNA synthetase. Biol Chem Hoppe Seyler 377:343-356.

Giuditta A, Kaplan BB, van Minnen J, Alvarez J, Koenig E (2002) Axonal and presynaptic protein synthesis: new insights into the biology of the neuron. Trends Neurosci 25:400-404.

Green ED, Birren B, Klapholz S, Myers RM, Riethman H, Roskams J (1999) Genome analysis: a laboratory manual. Cold Spring Harbor, NY: Cold Spring Harbor Laboratory.

Han JM, Kim JY, Kim S (2003) Molecular network and functional implications of macromolecular tRNA synthetase complex. Biochem Biophys Res Commun 303:985-993.

Hirakata M, Mimori T, Akizuki M, Craft J, Hardin JA, Homma M (1992) Autoantibodies to small nuclear and cytoplasmic ribonucleoproteins in Japanese patients with inflammatory muscle disease. Arthritis Rheum 35:449-456.

Ionasescu V, Searby C, Sheffield VC, Roklina T, Nishimura D, Ionasescu R (1996) Autosomal dominant Charcot-Marie-Tooth axonal neuropathy mapped on chromosome 7p (CMT2D). Hum Mol Genet 5:1373-1375.

Jordanova A, Irobi J, Thomas FP, Van Dijck P, Meerschaert K, Dewil M, Dierick I, Jacobs A, De Vriendt E, Guergueltcheva V, Rao CV, Tournev I, Gondim FA, D’Hooghe M, Van Gerwen V, Callaerts P, Van Den Bosch L, Timmermans JP, Robberecht W, Gettemans J, et al. (2006) Disrupted function and axonal distribution of mutant tyrosyl-tRNA synthetase in dominant intermediate Charcot-Marie-Tooth neuropathy. Nat Genet 38:197-202.

Kim JY, Kang YS, Lee JW, Kim HJ, Ahn YH, Park H, Ko YG, Kim S (2002) p38 is essential for the assembly and stability of macromolecular tRNA synthetase complex: implications for its physiological significance. Proc Natl Acad Sci USA 99:7912-7916.

Ko HS, von Coelln R, Sriram SR, Kim SW, Chung KK, Pletnikova O, Troncoso J, Johnson B, Saffary R, Goh EL, Song H, Park BJ, Kim MJ, Kim S, Dawson VL, Dawson TM (2005) Accumulation of the authentic parkin substrate aminoacyl-tRNA synthetase cofactor, p38/JTV-1, leads to catecholaminergic cell death. J Neurosci 25:7968-7978.

Ko YG, Kang YS, Kim EK, Park SG, Kim S (2000) Nucleolar localization of human methionyl-tRNA synthetase and its role in ribosomal RNA synthesis. J Cell Biol 149:567-574.

Liu Q, Dreyfuss G (1996) A novel nuclear structure containing the survival of motor neurons protein. EMBO J 15:3555-3565.

Mersiyanova IV, Perepelov AV, Polyakov AV, Sitnikov VF, Dadali EL, Oparin RB, Petrin AN, Evgrafov OV (2000) A new variant of Charcot-MarieTooth disease type 2 is probably the result of a mutation in the neurofilament-light gene. Am J Hum Genet 67:37-46.

Paratcha G, Ledda F, Baars L, Coulpier M, Besset V, Anders J, Scott R, Ibanez 
CF (2001) Released GFRalpha1 potentiates downstream signaling, neuronal survival, and differentiation via a novel mechanism of recruitment of c-Ret to lipid rafts. Neuron 29:171-184.

Ross RA, Spengler BA, Biedler JL (1983) Coordinate morphological and biochemical interconversion of human neuroblastoma cells. J Natl Cancer Inst 71:741-747.

Salazar-Grueso EF, Kim S, Kim H (1991) Embryonic mouse spinal cord motor neuron hybrid cells. NeuroReport 2:505-508.

Sambuughin N, Sivakumar K, Selenge B, Lee HS, Friedlich D, Baasanjav D, Dalakas MC, Goldfarb LG (1998) Autosomal dominant distal spinal muscular atrophy type V (dSMA-V) and Charcot-Marie-Tooth disease type 2D (CMT2D) segregate within a single large kindred and map to a refined region on chromosome 7p15. J Neurol Sci 161:23-28.

Sharma M, Sharma P, Pant HC (1999) CDK-5-mediated neurofilament phosphorylation in SHSY5Y human neuroblastoma cells. J Neurochem 73:79-86

Shiba K, Schimmel P, Motegi H, Noda T (1994) Human glycyl-tRNA synthetase. Wide divergence of primary structure from bacterial counterpart and species-specific aminoacylation. J Biol Chem 269:30049-30055.

Sikorski RS, Hieter P (1989) A system of shuttle vectors and yeast host strains designed for efficient manipulation of DNA in Saccharomyces cerevisiae. Genetics 122:19-27.

Sivakumar K, Kyriakides T, Puls I, Nicholson GA, Funalot B, Antonellis A, Sambuughin N, Christodoulou K, Beggs JL, Zamba-Papanicolaou E, Ionasescu V, Dalakas MC, Green ED, Fischbeck KH, Goldfarb LG (2005)
Phenotypic spectrum of disorders associated with glycyl-tRNA synthetase mutations. Brain 128:2304-2314.

Skre H (1974) Genetic and clinical aspects of Charcot-Marie-Tooth's disease. Clin Genet 6:98-118.

Targoff IN (1990) Autoantibodies to aminoacyl-transfer RNA synthetases for isoleucine and glycine. Two additional synthetases are antigenic in myositis. J Immunol 144:1737-1743.

Turner RJ, Lovato M, Schimmel P (2000) One of two genes encoding glycyltRNA synthetase in Saccharomyces cerevisiae provides mitochondrial and cytoplasmic functions. J Biol Chem 275:27681-27688.

Verhoeven K, De Jonghe P, Coen K, Verpoorten N, Auer-Grumbach M, Kwon JM, FitzPatrick D, Schmedding E, De Vriendt E, Jacobs A, Van Gerwen V, Wagner K, Hartung HP, Timmerman V (2003) Mutations in the small GTP-ase late endosomal protein RAB7 cause Charcot-MarieTooth type 2B neuropathy. Am J Hum Genet 72:722-727.

Williams J, Osvath S, Khong TF, Pearse M, Power D (1995) Cloning, sequencing and bacterial expression of human glycine tRNA synthetase. Nucleic Acids Res 23:1307-1310.

Wood JD, Beaujeux TP, Shaw PJ (2003) Protein aggregation in motor neurone disorders. Neuropathol Appl Neurobiol 29:529-545.

Zhao C, Takita J, Tanaka Y, Setou M, Nakagawa T, Takeda S, Yang HW, Terada S, Nakata T, Takei Y, Saito M, Tsuji S, Hayashi Y, Hirokawa N (2001) Charcot-Marie-Tooth disease type $2 \mathrm{~A}$ caused by mutation in a microtubule motor KIF1Bbeta. Cell 105:587-597. 